\title{
APPROXIMATING FIXED POINTS BY ISHIKAWA ITERATES
}

\author{
M. MaIti AND M.K. Ghosh
}

In a uniformly convex Banach space the convergence of Ishikawa iterates to a fixed point is discussed for nonexpansive and generalised nonexpansive mappings.

\section{INTRODUCTION}

Senter and Dotson [6] have given conditions under which a Mann type iterative process $[5]$ generated by a nonexpansive mapping in a uniformly convex Banach space converges to a fixed point of the mapping. In this paper we modify these conditions so that an Ishikawa iterative process [4] for a nonexpansive mapping in a uniformly convex Banach space converges to a fixed point of the mapping under these modified conditions. To achieve this we follow closely the analysis given in [6]. Then we show that these conditions are satisfied by generalised nonexpansive mappings, implying convergence of Ishikawa iterates for these mappings without any precondition.

\section{Preliminaries}

Let $X$ be a Banach space and $C$ a convex subset of $X$. A mapping $T: C \rightarrow C$ is said to be nonexpansive if $\|T x-T y\| \leqslant\|x-y\|$ for all $x, y \in C$. A mapping $T: C \rightarrow$ $C$ is said to be quasi-nonexpansive if $T$ has a fixed point $p \in C$ such that $\|T x-p\| \leqslant$ $\|x-p\|$ for all $x \in C$. The concept of quasi-nonexpansiveness is more general than that of nonexpansiveness. Indeed, a nonexpansive mapping with at least one fixed point is quasi-nonexpansive, but there exist quasi-nonexpansive mappings which are not nonexpansive. A mapping $T: C \rightarrow C$ is said to be generalised nonexpansive if

$$
\|T x-T y\| \leqslant a\|x-y\|+b\{\|x-T x\|+\|y-T y\|\}+c\{\|x-T y\|+\|y-T x\|\}
$$

for all $x, y \in C$, where $a, b, c \geqslant 0$ with $a+2 b+2 c \leqslant 1$. With further restrictions on $a$, $b, c$ it can be shown that a generalised nonexpansive mapping is also quasi-nonexpansive if it has a fixed point.

We now state the following conditions:

Received 7 October 1988

Copyright Clearance Centre, Inc. Serial-fee code: 0004-9729/89 \$A2.00+0.00. 
Condition A. A mapping $T: C \rightarrow C$ with nonempty fixed point set $F$ is said to satisfy Condition $\mathrm{A}$ if there is a nondecreasing function $f:[0, \infty) \rightarrow[0, \infty)$ with $f(0)=0$ and $f(r)>0$ for all $r \in(0, \infty)$ such that $\|x-T y\| \geqslant f(d(x, F))$ for $x \in C$ and all corresponding $y=(1-t) x+t T x$, where $0 \leqslant t \leqslant \beta<1$ and $d(x, F)=$ $\inf _{z \in F}\|x-z\|$.

Condition B. A mapping $T: C \rightarrow C$ with nonempty fixed point set $F$ is said to satisfy Condition $\mathrm{B}$ if there exists a real number $k>0$ such that $\|x-T y\| \geqslant k d(x, F)$ for $x \in C$ and all corresponding $y=(1-t) x+t T x$ where $0 \leqslant t \leqslant \beta<1$.

It may be noted that the mappings which satisfy Condition $B$ also satisfy Condition A. However, Condition B may be verified easily in examples. We also note that Conditions I and II of Senter and Dotson [6] are identical with Conditions A and B when $t=0$.

\section{ISHIKAWA ITERATIVE PROCESS}

Let $X$ be a Banach space $X$ and $C$ a convex subset of $X$. For $x_{1} \in C$, define a sequence $\left\{x_{n}\right\}_{n=1}^{\infty}$ such that

$$
x_{n+1}=\left(1-\alpha_{n}\right) x_{n}+\alpha_{n} T\left[\left(1-\beta_{n}\right) x_{n}+\beta_{n} T x_{n}\right]
$$

where $\left\{\alpha_{n}\right\}_{n=1}^{\infty}$ and $\left\{\beta_{n}\right\}_{n=1}^{\infty}$ are sequences of positive numbers. Ishikawa [4] has introduced this iteration scheme and discussed its convergence in the case of Lipschitzian pseudocontractive mappings imposing some restrictions on $\alpha_{n}$ and $\beta_{n}$. However, in the present paper we restrict them to satisfy

$$
\text { (i) } 0<a \leqslant \alpha_{n} \leqslant b<1 \text {, (ii) } 0 \leqslant \beta_{n} \leqslant \beta<1 \text {. }
$$

We denote by $M\left(x_{1}, \alpha_{n}, \beta_{n}, T\right)$ the sequence (1) with restriction (2).

We note that Senter and Dotson [6] have discussed the convergence of a particular case of sequence (1) by taking $\beta_{n}=0$ and $\alpha_{n}$ to satisfy (2) (i) for all $n$.

Before we discuss our results we recall the following lemma due to Dotson [2].

LEMMA. If $\left\{s_{n}\right\}$ and $\left\{t_{n}\right\}$ are sequences in the closed unit ball of a uniformly convex Banach space and if $\left\{z_{n}\right\}=\left\{\left(1-\alpha_{n}\right) s_{n}+\alpha_{n} t_{n}\right\}$ satisfies $\lim _{n \rightarrow \infty}\left\|z_{n}\right\|=1$, where $0<a \leqslant \alpha_{n} \leqslant b<1$, then $\lim _{n \rightarrow \infty}\left\|s_{n}-t_{n}\right\|=0$.

Theorem 1. Let $C$ be a nonempty, closed and convex subset of a uniformly convex Banach space $X$ and $T$ a quasi-nonexpansive mapping of $C$ into itself. If $T$ satisfies Condition $A$, where $F$ is the fixed point set of $T$ in $C$, then, for arbitrary $x_{1} \in C$, the sequence $M\left(x_{1}, \alpha_{n}, \beta_{n}, T\right)$ converges to a member of $F$. 
PRoof: If $x_{1} \in F$, then the result is trivial. We assume $x_{1} \in C \backslash F$. Now setting $y_{n}=\left(1-\beta_{n}\right) x_{n}+\beta_{n} T x_{n}$, we have for an arbitrary $z \in F$

$$
\begin{aligned}
\left\|x_{n+1}-z\right\| & =\left\|\left(1-\alpha_{n}\right)\left(x_{n}-z\right)+\alpha_{n}\left(T y_{n}-z\right)\right\| \\
& \leqslant\left(1-\alpha_{n}\right)\left\|x_{n}-z\right\|+\alpha_{n}\left\|y_{n}-z\right\| \\
& =\left(1-\alpha_{n}\right)\left\|x_{n}-z\right\|+\alpha_{n}\left\|\left(1-\beta_{n}\right)\left(x_{n}-z\right)+\beta_{n}\left(T x_{n}-z\right)\right\| \\
& \leqslant\left(1-\alpha_{n}\right)\left\|x_{n}-z\right\|+\alpha_{n}\left(1-\beta_{n}\right)\left\|x_{n}-z\right\|+\alpha_{n} \beta_{n}\left\|x_{n}-z\right\| \\
& =\left\|x_{n}-z\right\| .
\end{aligned}
$$

This implies that $d\left(x_{n+1}, F\right) \leqslant d\left(x_{n}, F\right)$ and hence that the sequence $\left\{d\left(x_{n}, F\right)\right\}$ is nonincreasing. Further, it is bounded below. Thus $\lim _{n \rightarrow \infty} d\left(x_{n}, F\right)$ exists. Next, we show that this limit is zero.

Suppose that $\lim _{n \rightarrow \infty} d\left(x_{n}, F\right)=b>0$. Then, for some $z_{0} \in F, \lim _{n \rightarrow \infty}\left\|x_{n}-z_{0}\right\|=$ $b^{\prime}>b>0$. Choose a positive integer $N$ such that $\left\|x_{n}-z_{0}\right\| \leqslant 2 b^{\prime}$ for $n \geqslant N$. If we now set $s_{n}=\left(x_{n}-z_{0}\right) /\left\|x_{n}-z_{0}\right\|$ and $t_{n}=\left(T y_{n}-z_{0}\right) /\left\|x_{n}-z_{0}\right\|$, then we observe that $\left\|s_{n}\right\|=1$ and $\left\|t_{n}\right\| \leqslant 1$ for all $n$. Further, $\left\|\left(1-\alpha_{n}\right) s_{n}+\alpha_{n} t_{n}\right\|=$ $\left\|\left(1-\alpha_{n}\right) x_{n}+\alpha_{n} T y_{n}-z_{0}\right\| /\left\|x_{n}-z_{0}\right\|=\left\|x_{n+1}-z_{0}\right\| /\left\|x_{n}-z_{0}\right\| \rightarrow 1$ as $n \rightarrow \infty$. But, for $n \geqslant N$, we have

$$
\left\|s_{n}-t_{n}\right\|=\frac{\left\|x_{n}-T y_{n}\right\|}{\left\|x_{n}-z_{0}\right\|} \geqslant \frac{f\left(d\left(x_{n}, F\right)\right)}{\left\|x_{n}-z_{0}\right\|} \geqslant \frac{f(b)}{2 b^{\prime}}>0
$$

implying $\lim _{n \rightarrow \infty}\left\|s_{n}-t_{n}\right\| \neq 0$, which contradicts the result of the lemma. Hence $\lim _{n \rightarrow \infty} d\left(x_{n}, F\right)=0$.

Now, following the same arguments of Senter and Dotson [6], which we omit here in order to avoid repetition, it may be shown that the sequence $M\left(x_{1}, \alpha_{n}, \beta_{n}, T\right)$ converges to a member of $F$.

Bose and Mukherjee [1] have discussed the convergence of Mann type iteration schemes generated by generalised nonexpansive mappings. In the sequel we discuss the convergence of Ishikawa iteration scheme when the mappings are generalised nonexpansive.

Theorem 2. Let $C$ be a nonempty, bounded, closed and convex subset of a uniformly convex Banach space $X$, and $T: C \rightarrow C$ be a continuous mapping such that

$$
\|T x-T y\| \leqslant a\|x-y\|+b\{\|x-T x\|+\|y-T y\|\}+c\left\{\|x-T y\|_{\lambda}+\|y-T x\|_{\lambda}\right.
$$

for all $x, y \in C$, where $a, c \geqslant 0$ and $b>0$ with $a+2 b+2 c \leqslant 1$. Then, for arbitrary $x_{1} \in C$, the sequence $M\left(x_{1}, \alpha_{n}, \beta_{n}, T\right)$ converges to the unique fixed point of $T$. 
Proof: By Theorem 2 of Goebel, Kirk and Shimi [3] the mapping $T$ has a fixed point, which is also unique because of the additional restriction on $b$. Let $p$ be the fixed point. Then, setting $y=p$ in (3), we have

$$
\begin{aligned}
\|T x-p\| & \leqslant(a+c)\|x-p\|+b\|x-T x\|+c\|T x-p\| \\
& \leqslant(a+b+c)\|x-p\|+(b+c)\|T x-p\|
\end{aligned}
$$

implying

$$
\|T x-p\| \leqslant \frac{a+b+c}{1-b-c}\|x-p\| \leqslant\|x-p\| .
$$

Thus $T$ is quasi-nonexpansive.

For any $y \in C$ we observe from (4) that

$$
\|T y-p\| \leqslant(a+c)\|y-p\|+b\|y-T y\|+c\|T y-p\| \text {. }
$$

Now, set $y=(1-t) x+t T x$, where $0 \leqslant t \leqslant \beta<1$. Then

$$
\|y-p\| \leqslant\|x-p\|,\|y-x\|=t\|x-T x\| \leqslant 2 t\|x-p\|
$$

whence we get

$$
\begin{aligned}
\|T y-p\| & \leqslant(a+c)\|y-p\|+c\|x-p\|+b\|y-x\|+(b+c)\|x-T y\| \\
& \leqslant(a+2 c)\|x-p\|+b\|y-x\|+(b+c)\|x-T y\| .
\end{aligned}
$$

Also it is obvious that

$$
\|T y-p\| \geqslant\|x-p\|-\|x-T y\|
$$

Combining (6) and (7) we derive

$$
b\|y-x\|+(1+b+c)\|x-T y\| \geqslant(1-a-2 c)\|x-p\| \geqslant 2 b\|x-p\|,
$$

whence, by applying the second inequality in (5), we finally derive

$$
\|x-T y\| \geqslant \frac{2 b(1-t)}{1+b+c}\|x-p\| \geqslant \frac{2 b(1-\beta)}{1+b+c}\|x-p\|
$$

where $2 b(1-\beta) /(1+b+c)>0$. Thus $T$ satisfies Condition $\mathrm{B}$ and hence Condition A. Then the result follows from Theorem 1 .

A variant of Theorem 2 may be stated in the following form. 
Theorem 3. Let $X$ be a uniformly convex Banach space and $C$ a nonempty, bounded, closed and convex subset of $X$. If $T: C \rightarrow C$ is a mapping satisfying

$$
\|T x-T y\| \leqslant a\|x-y\|+b\{\|x-T x\|+\|y-T y\|\}+c\{\|x-T y\|+\|y-T x\|\}
$$

for all $x, y \in C$, where $a, b, c \geqslant 0$ with $3 a+2 b+4 c \leqslant 1$, then, for arbitrary $x_{1} \in C$, the sequence $M\left(x_{1}, \alpha_{n}, \beta_{n}, T\right)$ converges to the unique fixed point of $T$.

In this case the existence of a fixed point is ensured by the fact that $C$ has a normal structure, see [1]. The fixed point is also unique. As in Theorem 2, it can be shown that $T$ is quasi-nonexpansive and satisfies Condition $B$.

Finally it may be remarked that Theorems 5 and 6 of Bose and Mukherjee [1] follow as particular cases of Theorems 2 and 3.

\section{REFERENCES}

[1] R.K. Bose and R.N. Mukherjee, 'Approximating fixed points of some mappings', Proc. Amer. Math. Soc. 82 (1981), 603-606.

[2] W.G. Dotson, Jr., 'On the Mann iterative process', Trans. Amer. Math. Soc. 149 (1970), 65-73.

[3] K. Goebel, W.A. Kirk and T.N. Shimi, 'A fixed point theorem in uniformly convex spaces', Boll. Un. Mat. Ital. 7 (1973), 67-75.

[4] S. Ishikawa, 'Fixed points by a new iteration method', Proc. Amer. Math. Soc. 44 (1974), $147-150$.

[5] W.R. Mann, 'Mean value methods in iteration', Proc. Amer. Math. Soc. 4 (1953), 506-510.

[6] H.F. Senter and W.G. Dotson, Jr., 'Approximating fixed points of nonexpansive mappings', Proc. Amer. Math. Soc. 44 (1974), 375-380.

M. Maiti

Department of Mathematics

Indian Institute of Technology

Kharagpur - 721302

India
M.K. Ghosh

Departinent of Mathematics

Narajole Raj College

Midnapore

India 